\title{
SUBSTORMS AT JUPITER: GALILEO OBSERVATIONS OF TRANSIENT RECONNECTION IN THE NEAR TAIL
}

\author{
C. T. Russell ${ }^{1,2}$, K. K. Khurana ${ }^{1}$, M. G. Kivelson ${ }^{1,2}$ and D. E. Huddleston ${ }^{1}$ \\ ${ }^{I}$ Institute of Geophysics and Planetary Physics, University of California, \\ Los Angeles, CA 90095-1567, USA \\ ${ }^{2}$ Department of Earth and Space Sciences, University of California, \\ Los Angeles, CA 90095-1567, USA
}

\begin{abstract}
The magnetic flux content of the Jovian magnetosphere is set by the internal dynamo, but those magnetic field lines are constantly being loaded by heavy ions at the orbit of Io and dragged inexorably outward by the centrifugal force. Vasyliunas has proposed a steady state reconnecting magnetospheric model that sheds plasma islands of zero net magnetic flux and returns nearly empty flux tubes to the inner magnetosphere. The Galileo observations indicate that beyond $40 R_{J}$ the current sheet begins to tear and beyond $50 \mathrm{R}_{\mathrm{J}}$ on the nightside explosively reconnects as the tearing site reaches the low density lobe region above and below the current sheet. Small events occur irregularly but on average about every 4 hours and large events about once a day. The magnetic flux reconnected in such events amounts up to about 70,000 Webers/sec and is sufficient to return the outwardly convected magnetic flux to the inner magnetosphere. Since this process releases plasmoids into the jovian tail, as do terrestrial substorms; since this process involves explosive reconnection across the current sheet on the nightside of the planet, as do terrestrial substorms; and since the process is a key in closing the circulation pattern of the magnetic and plasma flux, as it is in terrestrial substorms; we refer to these events as jovian substorms.
\end{abstract}

(c) 2000 COSPAR. Published by Elsevier Science Ltd. All rights reserved.

\section{INTRODUCTION}

Pioneer 10 and 11, Voyager 1 and 2, and Ulysses observations revealed that the jovian magnetosphere had an embedded current disk; a magnetotail-like current sheet at all local times [Smith et al., 1975; Ness et al., 1979; Balogh et al., 1992]. The cause of the stretched configuration was the centrifugal pressure forces of the plasma added to magnetospheric field line at Io. The rate of mass addition has been estimated to be as high as 1 ton/second [Hill et al., 1983]. The mass in the Io torus cannot simply build up forever. It must be lost from the system at a roughly constant rate that matches the addition at Io. The current paradigm for this loss process is that the plasma convects outward extracting angular momentum from the ionosphere to maintain its rotation rate near that of the ionosphere. At some distance on the nightside the stretched-out field reconnects, ejecting ions down the tail and returning magnetic flux to the inner magnetosphere [Vasyliunas, 1983]. The Galileo data confirm this general picture and show that the process is not steady or periodic but rather stocastic. They show where in local time and radial distance the reconnection occurs and the explosive nature of that reconnection. In this paper we examine the structure of the current sheet beyond $40 R_{J}$ 


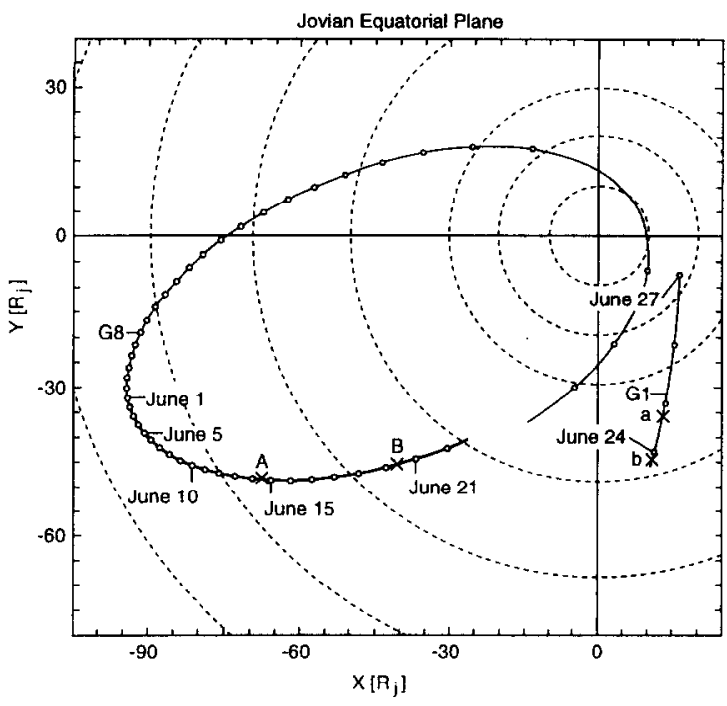

Figure 1. The orbit of the Galileo spacecraft in the jovian equator. All of the eighth orbit G8 and the part of the first orbit, G1, relevant to the analysis herein are shown. The letters $a$ and $b$ show where the data in Figures 2 and 3 were obtained. The thick line shows the region in which the data of Figure 5 were obtained. The letters A and B show where the data in Figures 6 and 7 were obtained.

and provide evidence for the transient reconnection of the field across the current sheet beyond $50 \mathrm{R}_{\mathrm{J}}$. We will examine data from the inbound pass of the first Galileo orbit, G1, and the eighth orbit G8. A portion of the first orbit and most of the latter orbit are shown in Figure 1. The location of the events to be examined herein are shown with $\mathrm{X}$ 's.

\section{STRUCTURE OF THE CURRENT SHEET, 25-50 $\mathrm{R}_{\mathrm{J}}$}

Figure 2 shows time series of the magnetic field during a current sheet crossing at $39.1 \mathrm{R}_{\mathrm{J}}$ and $0717 \mathrm{LT}$ expressed in the minimum variance coordinate system obtained using low pass filtered data that exhibited only the large scale variation of the field, the discontinuity structure. This point is marked ' $a$ ' in Figure 1. On top of this large-scale structure is small-scale structure that can be seen as noise in the normal component. In the radial range from 25 to $40 \mathrm{R}_{\mathrm{J}}$ this structure is smaller than the average normal component across the sheet so the normal component does not reverse. There is structure in the current sheet but there is no evidence for tearing of that current sheet into magnetic islands.

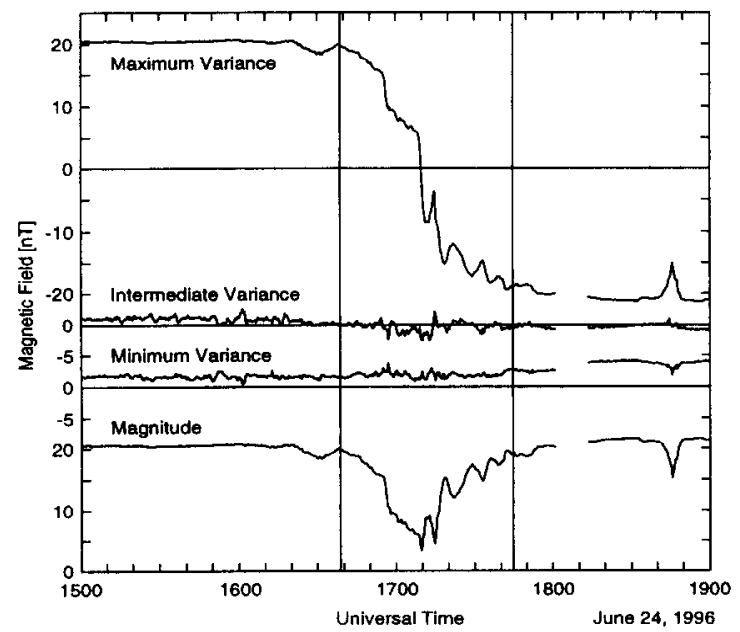

Figure 2. Magnetic field measurements through the magnetodisk current sheet at $39.1 \mathrm{R}_{\mathrm{J}}$ and $0717 \mathrm{LT}$. The measurements have been rotated into a principal axis system based on low pass filtered data. 


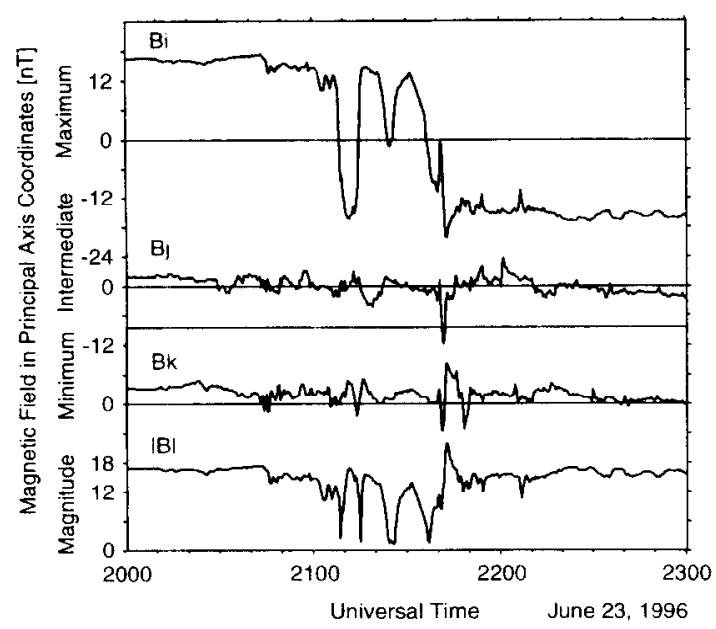

Figure 3. Magnetic field measurements through the magnetodisk current sheet at $46 \mathrm{R}_{\mathrm{J}}$ and $0653 \mathrm{LT}$. The measurements have been rotated into a principal axis system based on low pass filtered data.

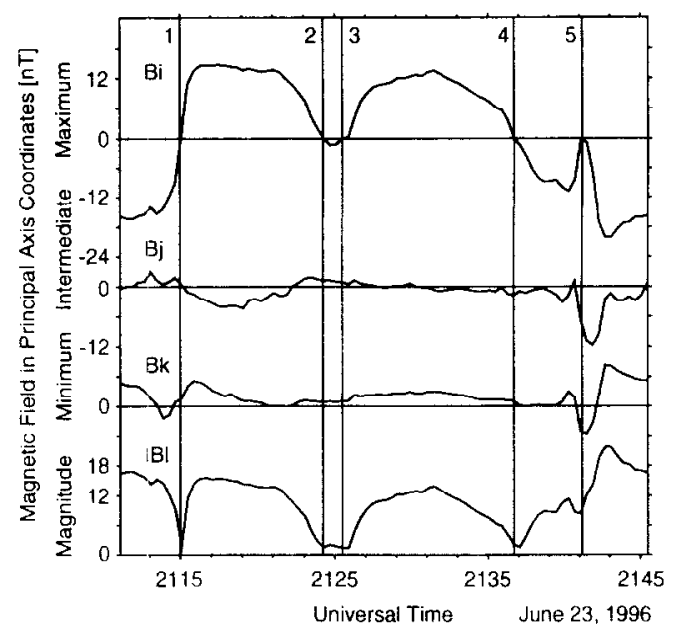

Figure 4. The central portion of Figure 3 cxpanded to show the current sheet crossing in greater resolution.

Figure 3 shows a current sheet crossing in the same format at $47 \mathrm{R}_{\mathrm{J}}$ and $0653 \mathrm{LT}$ on the same pass at point ' $b$ ' on Figure 1. Here there are multiple crossings of the current

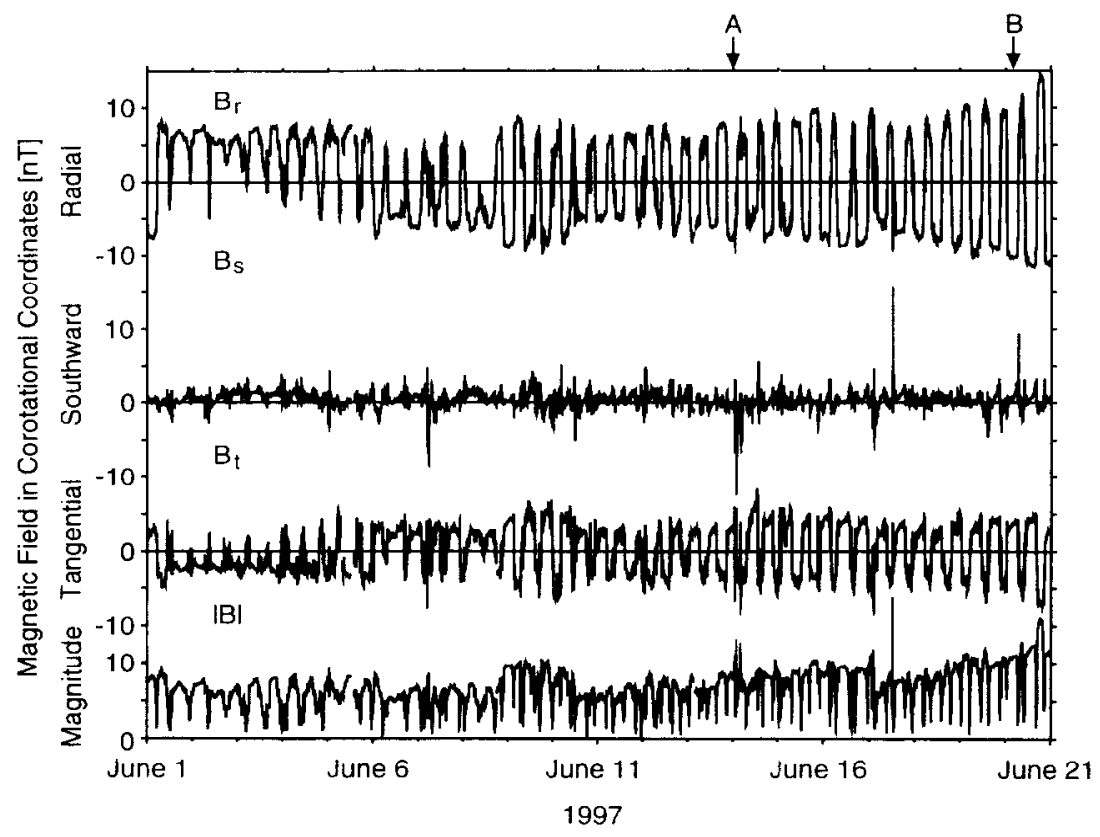

Figure 5. One minute time resolution magnetic field measurements from June 1 to June 21, 1997 on Galileo's eighth orbit of Jupiter in the radial, southward and tangential (rst) coordinate system. The spacecraft moves from a radial distance of $100 R_{\mathrm{J}}$ and a local time of 0116 to a radial distance of $58 \mathrm{R}_{\mathrm{J}}$ and a local time of 0324 . 


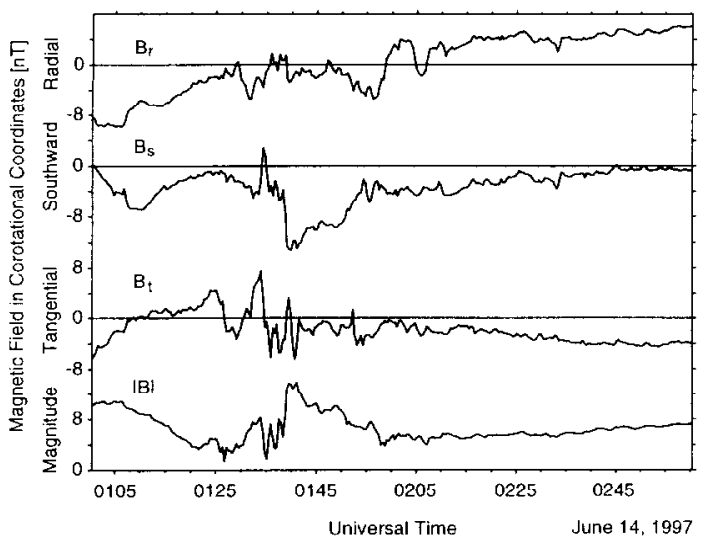

Figure 6. High resolution display of a transient reconfiguration of the current sheet on June 14, 1997 from 0100 to $0300 \mathrm{UT}$ at $84.8 \mathrm{R}_{\mathrm{J}}$ and $0220 \mathrm{LT}$.

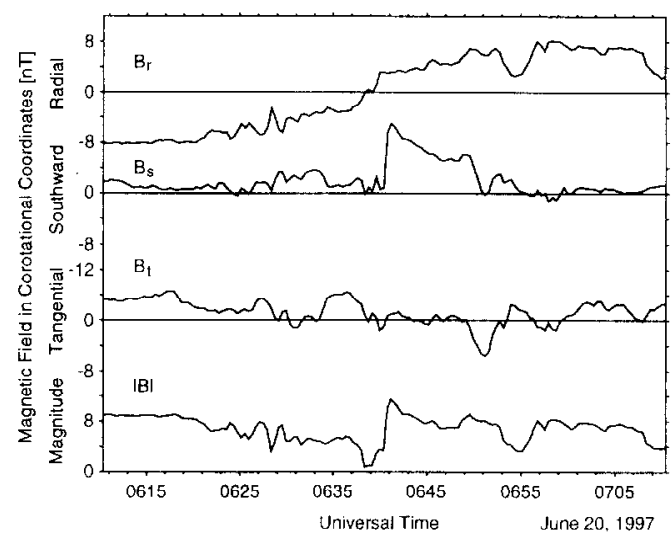

Figure 7. High resolution display of a transient reconfiguration of the current sheet on June 20, 1997 from 0610 to $0710 \mathrm{UT}$ at $63.2 \mathrm{R}_{\mathrm{J}}$ and $0316 \mathrm{LT}$.

sheet. Also here the high frequency noise is larger than the average normal component in the vicinity of the current sheet traversal, and significantly so. The $B_{k}$ component has several intervals of negative values. Nevertheless, it is not clear that each of these is due to a tearing island. Figure 4 shows an expansion of these data with the current sheet crossings indicated. In fact at crossings 1 to 4 , the magnetic field is always positive in the direction of Jupiter's dipole field. Only at crossing 5, that is a very close approach to the current sheet rather than a crossing, is the normal component really negative. The other negative components at 2114 UT occurs away from the current sheet center and could be caused by a bending of the current sheet. The 2141 UT event cannot be so interpreted, so it appears that some of the structure here occurs when field lines become increasingly stretched so that they begin to tear but at these distances nothing dramatic happens.

\section{TRANSIENT RECONNECTION 50-100 $\mathrm{R}_{\mathrm{J}}$}

Figure 5 shows the Galileo magnetic field in radial, southward and tangential (or corotational), rst, coordinates from June 1 to June 21,1997 when it moved from a local time of 0116 and a distance of 100 $\mathrm{R}_{\mathrm{J}}$ through apojove and back to a distance of $58 \mathrm{R}_{\mathrm{J}}$ at a local time of 0324 . Of immediate notice are the 10-hour periodicities in $B_{r}$ and $B_{t}$ and the irregular transients in $B_{s}$. The $B_{s}$ transients are not telemetry noise but are real physical events as shown in the next two figures. We note that there is no obvious periodicity to these transients, but on average they recur about every 4 hours for events that cause a $2 \mathrm{nT}$ increase or decrease in Bs.

Figure 6 shows a high resolution plot of the magnetic field during the event marked $A$ in Figure 5 . This event is neither the largest nor the smallest of the events seen. The magnetic field turns strongly northward (negative $\mathrm{B}_{\mathrm{s}}$ ) during this event and the magnetic field strengthens.

Figure 7 shows a second typical event, marked B in Figure 5. This event has a southward turning of the $\mathrm{B}_{\mathrm{s}}$ component. Such turnings (both southward and northward) occur about once every 4 hours. Larger ones above 5 nT occur about once a day in this region ( $>50 \mathrm{R}_{\mathrm{J}}$ and from midnight to 3 AM local timc). For events such as these two that occurred while the spacecraft who close to the center of the current sheet it is difficult to tell whether a growth phase preceded the sudden change in the north-south component. In other events such as those shown in Russell et al. [1998] there were precursory changes. These may 
simply mark the time of the onset of reconnection elsewhere. It is most likely that the exponential-like decay of the normal component, seen in both figures here but better displayed in the events seen in Russell et al. [1998], is not only the recovery phase but also serves as the growth phase as well.

\section{SUMMARY AND CONCLUSIONS}

Examination of the jovian current sheet beyond $40 \mathrm{R}_{\mathrm{J}}$ shows an evolution of its structure as a function of radius. Inside about $45 R_{\mathrm{J}}$ the current sheet is relatively quiet and fluctuations in the normal component crossing the sheet do not reverse its polarity. Beyond $45 \mathrm{R}_{\mathrm{J}}$, the current sheet has structure in the normal component that reverses its polarity. We conjecture that this structure evolves only slowly in the relatively dense interior of the jovian current sheet. Although we only show results from the G1 pass here the G2 pass that has also been examined carefully show's the same behavior. We have not however undertaken this study at different local times.

On the nightside of the magnetodisk beyond $50 \mathrm{R}_{\mathrm{J}}$ the strength of the perturbation in the normal component increases so that periodically but approximately once every 4 hours the normal component rises above $2 \mathrm{nT}$, either northward or southward, and once a day, above $5 \mathrm{nT}$. These less frequent but much larger disturbances are interpreted as the jovian equivalent of terrestrial substorms. The largest events appear to take place explosively as they begin to reconnect magnetic field in the lobes above the magnetodisk where the density is low and the Alfven velocity is very high. The magnetic field can increase dramatically, over a factor of three, as the reconnected magnetic field piles up against the magnetodisk at closer distances. This process has much similarity to the terrestrial substorm with two exceptions. The normal component is not as enhanced above the lobe field strength in a terrestrial substorm and angular momentum does not play much of a role in the terrestrial case. However, at Jupiter angular momentum conservation can cause the "corotating" plasma to rotate even faster than the corotation velocity and the magnetic field can swing forward rather than be swept backward [Russell $e t$ al., 1998]. Another difference may be in the phases of a substorm. In the terrestrial case there is often a growth phase in which energy is stored in the tail from the solar wind. This is followed by expansion and recovery phases. At Jupiter there may be just two phases, expansion and recovery, with the recovery phase leading to the next expansion.

If we take the duration of the largest events and their normal components and assume that the velocity is half of that of corotation, then the largest events contain about $10^{10}$ Webers. Typical events reconnecting about $10^{9}$ Webers and at a rate of once every 4 hours can return about 70,000 Webers per second to the inner magnetosphere. This rate is sufficient to return the magnetic flux carried outward with the iogenic plasma. These results do not by themselves show how the magnetic flux makes its way from the midnight to $3 \mathrm{AM}$ sector and $50 \mathrm{R}_{\mathrm{J}}$ to the vicinity of Io but the presence of empty flux tubes in the Io torus suggests that the larger region of reconnected magnetic flux breaks into small filaments containing a few hundred Webers each on average and that the slender empty tubes rapidly float inward toward lo [Russell et al., 1999]. In summary these near tail reconnection events play a very similar role to the reconnection cvents in the Earth's tail. Thus, we conclude that these events are the jovian counterpart of terrestrial substorms even though they may be driven by internal processes and not by the solar wind.

\section{ACKNOWLEDGMENTS}

This work was supported by the National Aeronautics and Space Administration research grant through the Jet Propulsion Laboratory. 


\section{REFERENCES}

Balogh, A., M. K. Dougherty, R. J. Forsyth, D. J. Southwood, B. T. Tsurutani, et al., Magnetic field observations on the Ulysses flyby of Jupiter, Science, 257, 1515-1518 (1992).

Hill, T. W., A. J. Dessler, and C. K. Goertz, Magnetospheric models, in Physics of the Jovian Magnetosphere, edited by A.J. Dessler, pp. 353-394, Cambridge Univ. Press, New York, (1983).

Ness, N. F., M. H. Acuna, R. P. Lepping, L. F. Burlaga, K. W. Behannon, et al., Magnetic field studies at Jupiter by Voyager 1: Preliminary results, Science, 204 (4396), 982-987, (1979).

Russell, C. T., K. K. Khurana, D. E. Huddleston, and M. G. Kivelson, Localized reconnection in the near Jovian magnetotail, Science, 280, 1061-1064, (1998).

Russell, C. T., M. G. Kivelson, K. K. Khurana, and D. E. Huddleston, Circulation and dynamics in the jovian magnetosphere, Adv. Space Res., (1999).

Smith, E. J., B. V. Connor, and J. G. J. Foster, Measuring the magnetic fields of Jupiter, IEEE Trans. Magnetics, 11, 962-980, (1975).

Vasyliunas, V. M., Plasma distribution and flow, in Physics of the Jovian Magnetosphere, edited by A. J. Dessler, pp. 395-453, Cambridge University Press, London, (1983). 